\title{
DesarRollo DE PROVEEDORES LOCALES EN EL SECTOR EXTRACTIVO COLOMBIANO: CUELLOS DE BOTELLA, FACTORES DE ÉXITO E IMPLICACIONES DE POLÍTICA PÚBLICA
}

Juan David Gutiérrez-Rodríguez

\section{Resumen}

Las sociedades que cuentan con abundantes recursos naturales no renovables pueden transformar dicho capital natural en otros tipos de capital (p. ej. humano, producido, infraestructura) a través de diferentes canales. Para los gobiernos que deben administrar la riqueza de los recursos naturales, la sustitución de un tipo de capital -que se agota a medida que se explota- por otros capitales sostenibles, es un reto de política pública. Uno de los canales de transformación lo constituye la creación de encadenamientos productivos -"hacia adelante" y "hacia atrás"- entre el sector extractivo y otros sectores económicos. Este artículo tiene dos objetivos: i) identificar los principales cuellos de botella y factores de éxito para el desarrollo de proveedores nacionales y locales para el sector extractivo colombiano y ii) las implicaciones de política pública relacionadas con la promoción de encadenamientos productivos entre los sectores mineros y de hidrocarburos, y otros sectores en Colombia.

Palabras clave: sector extractivo, proveeduría local, contenido local, encadenamientos productivos, Colombia.

* Candidato a Doctor en Política pública, Blavatnik School of Government, Oxford University, Oxford (Reino Unido). juan.gutierrezrodriguez@bsg.ox.ac.uk

Recibido: 15 de septiembre de 2015 / Modificado: 24 de octubre de 2015 / Aceptado: 30 de octubre de 2015.

Para citar este artículo

Gutiérrez-Rodríguez, J. D. (2015). Desarrollo de proveedores locales en el sector extractivo colombiano: cuellos de botella, factores de éxito e implicaciones de política pública. OPERA, 17, pp. 5-25. DOI: http://dx.doi. org/10.18601/16578651.n17.02 


\section{DEVELOPMENT OF LOCAL PROVIDERS IN THE COLOMBIAN EXTRACTIVE SECTOR: BOTTLENECKS, SUCCESS FACTORS AND POLICY IMPLICATIONS}

\section{Abstract}

Societies that have abundant non-renewable natural resources may transform such natural capital into other types of capital (e.g. human, produced, infrastructure) through different channels. For governments that manage natural wealth, the substitution of one type of capital, which depletes as it is exploited, for other sustainable capitals is a public policy challenge. One of the channels of transformation is the creation of forward and backward productive linkages between the extractive sector and other economic sectors. This article has two objectives: (i) identify the main bottlenecks and success factors for the development of national and local suppliers for the Colombian extractive sector and (ii) the policy implications related to the promotion of productive linkages between the mining and hydrocarbon sectors in Colombia.

Key words: Extractive sector, local supply, local content, productive linkages, Colombia

\section{INTRODUCCIÓN}

Las sociedades que cuentan con abundantes recursos naturales no renovables pueden transformar dicho capital natural en otros tipos de capital (p. ej. humano, producido, infraestructura). Esta aproximación fue formulada hace casi cuatro décadas por John M. Hartwick (1977), quien propuso invertir todas las rentas derivadas de la explotación de recursos agotables en nuevas formas de capital "reproducible". La propuesta, denominada por la literatura como la "regla de Hartwick", pretende resolver un asunto atinente tanto al desarrollo sostenible como a la equidad intergeneracional (World Bank, 2006).

La sustitución de un capital natural que se agota medida que se explota por otros capitales sostenibles es quizás el reto económico fundamental que deben enfrentar los gobiernos que administran su riqueza de recursos naturales (van der Ploeg y Venables, 2012). Es preciso recordar que al menos cincuenta países, en los que viven 1,4 billones de personas, dependen económicamente de la explotación de recursos naturales no renovables (p. 316).

Este trabajo estudia uno de los canales de transformación del capital natural: la creación de encadenamientos productivos "hacia adelante" y "hacia atrás" entre el sector extractivo y otros sectores económicos ${ }^{1}$. El documento identifica los principales cuellos de botella y factores de éxito para el desarrollo de proveedores nacionales y locales para el sector extractivo colombiano, y deriva de este análisis las respectivas implicaciones de política pública.

Si bien la revisión de literatura muestra que el tema de los encadenamientos de los

\footnotetext{
1 Otros canales de transformación, no abordados en este texto, son la inversión pública de las rentas mineroenergéticas y la misma actividad extractiva vía -por ejemplo- la generación de empleo, inversión en infraestructura y la formación de capital humano.
} 
sectores minero y petrolero colombiano ha sido estudiado, las investigaciones se limitan a su caracterización y a calcular su impacto en la economía (Reina y Cárdenas, 2008; Olivera, Zuleta, Aguilar y Orozco, 2011; Perry y Palacios, 2013; Martínez y Aguilar, 2013). Otros estudios, como el de Cadena y Pinzón (2011), presentan propuestas muy concretas para su promoción, como la estructuración de un "Programa nacional de proveedores y servicios”. Más recientemente, Castillo (2014) propuso una agenda de acción "público-privada" para promover una industria de proveedores minero-energéticos en el país enfocada a los departamentos con mayor volumen de inversión proyectada. Asimismo, Castillo (2014) propone una hoja de ruta para mejorar la competitividad de dichos departamentos.

Teniendo en cuenta el estado del arte sobre la materia, este artículo pretende ayudar a llenar una brecha de la literatura relacionada con los cuellos de botella que les impiden o inhiben o desincentivan a las empresas del sector extractivo realizar compras de servicios y bienes a empresas locales, así como los factores de éxito que inciden en la capacidad de las empresas nacionales de otros sectores para desarrollar y proveer efectivamente bienes y servicios al sector minero-energético.
El artículo se realizó bajo una metodología cualitativa que se nutrió de reportes oficiales, información publicada por las empresas y de entrevistas semiestructuradas a funcionarios de empresas de hidrocarburos y minería en Colombia y funcionarios del Gobierno nacional ${ }^{2}$. Asimismo, el documento utilizó literatura secundaria sobre el desarrollo de proveedores para el sector extractivo en Colombia. Esta literatura se complementa con bibliografía sobre casos en Perú, Chile y Brasil. Finalmente, el documento también usó como fuentes secundarias reportes de prensa.

El documento está dividido en cinco secciones, incluida esta. La segunda sección, explica por qué los encadenamientos productivos pueden constituir un mecanismo de generación de nuevos capitales. La tercera sección, presenta un balance del estado de los encadenamientos productivos en Colombia, con énfasis en los enlaces "hacia atrás" o "aguas arriba” ". La cuarta sección se enfoca en explicar los principales hallazgos sobre los obstáculos en la promoción de estos encadenamientos. Asimismo, se exponen los principales factores que la literatura existente ha identificado como incluyentes para el éxito del desarrollo de los encadenamientos entre empresas nacionales,

\footnotetext{
2 Las entrevistas se realizaron en su mayoría a mediados del año 2014, cuando los precios del petróleo aún no se habían desplomado. Sin embargo, algunas entrevistas se realizaron en el segundo semestre de 2015, cuando los efectos de la caída de los precios del petróleo empezaba a generar efectos en el sector. Las entrevistas incluyeron: i) funcionarios de Ecopetrol, Cerromatoso, AngloGold y Gramalote; ii) funcionarios de Asomineros-Andi y la Asociación Colombiana de Minería; y, iii) funcionarios del Ministerio de Minas y Energía, la Agencia Nacional Minera y la Unidad de Planeación Minero-Energética.

3 Uno de los limitantes para el desarrollo de encadenamientos "hacia adelante" con el sector minero es la limitada producción minera del país en el contexto internacional (Castillo, 2014).
} 
y empresas mineras y de hidrocarburos en Colombia.

Finalmente, en la última sección se presentan las implicaciones de política pública de lo expuesto en las anteriores secciones, con recomendaciones puntuales con responsables y condiciones para su éxito. Se advierte que, en la medida en que se asignan roles a diferentes actores (Gobierno, sector extractivo, proveedores), superar los retos identificados requiere mucho más que la formulación e implementación de políticas públicas: se requiere un trabajo estrecho entre agentes del sector privado (sector extractivo, gremios, proveedores) y entre el sector privado y el Estado como ocurre en Brasil, Canadá, Chile y Australia.

\section{IMPORTANCIA DE LOS ENCADENAMIENTOS PRODUCTIVOS NACIONALES Y LOCALES}

El impacto en el desarrollo económico de una región o país en virtud del desarrollo de proveedores de bienes y servicios locales para empresas mineras y de hidrocarburos es el principal motivo por el cual los gobiernos promueven estos encadenamientos. Precisamente, el desarrollo de estos encadenamientos es uno de los objetivos de las políticas de "contenido local" implementadas por los gobiernos en sus sectores extractivos. La definición sobre qué tipos de bienes o servicios pueden calificarse como "contenido local" varía en las diferentes legislaciones y políticas de los países. Para definir lo anterior, los Estados adoptan criterios relativos a la titularidad de la propiedad de las empresas que proveen los bienes o prestan los servicios, al lugar en el cual las empresas cuentan con presencia operacional, o al valor agregado local o nacional en el respectivo producto o servicio (Esteves, Coyne y Moreno, 2013 ). A pesar de la divergencia en los criterios para calificar el "contenido local", el punto común consiste en el grado en el que "la producción de la industria extractiva genera beneficios adicionales a la economía más allá de la contribución directa de su valor agregado, mediante sus vínculos con otros sectores" (Tordo, Warner, Manzano y Anouti, 2013, p. xi).

Consecuentemente, el objetivo de las políticas de contenido nacional o local consiste, por una parte, en "extender o expandir los beneficios de las actividades petroleras, gasíferas y mineras para la economía nacional” $y$, por la otra, en "brindarles a las partes interesadas locales acceso a oportunidades económicas, ya sea relacionadas con el empleo, la participación en cadenas de suministro o la provisión de servicios de apoyo relacionados" (Esteves, Coyne y Moreno, 2013, p. 2).

El impacto económico del sector extractivo no se limita a sus aportes en términos de producción, inversión directa (nacional y extranjera), exportaciones, divisas, impuestos y regalías, y empleos. Dicho sector tiene impactos directos e indirectos sobre otras actividades económicas. "Cualquier proceso productivo genera dinamismo en los sectores que le proporcionan insumos, así como en aquellos que utilizan sus productos en nuevos procesos de transformación" (Reina y Cárdenas, 2008, p. 21). Adicionalmente, la diversificación de la economía a través de la innovación, produc- 
ción y exportación ${ }^{4}$ de nuevos productos está estrechamente relacionada con el crecimiento económico de largo plazo (Gelb, 2011; Gylfason, 2011; Perry y Palacios, 2013).

En ese sentido, países como Brasil, Chile, México, Australia, Canadá y Noruega (entre otros), han promovido la generación de eslabonamientos (hacia adelante y hacia atrás) de sus sectores extractivos con otros sectores de la economía, logrando un mayor impacto de esta actividad en términos de innovación y empleo (Reina y Cárdenas, 2008; Gelb, 2011; Gylfason, 2011; Perry y Palacios 2013; Ryggvik, 2013; de Oliveira 2015). En el caso de Colombia, diversos investigadores (Reina y Cárdenas, 2008; Olivera, Zuleta, Aguilar y Orozco, 2011; Castro, Zuleta, Alandette, Fonseca y Martínez, 2012; Martínez y Aguilar, 2013; Perry y Palacios, 2013) señalan la importancia de contar con políticas públicas y programas empresariales que procuren una mayor agregación de valor agregado en la cadena minero-energética y el fortalecimiento de los encadenamientos entre este sector y el resto de la economía.

Por otra parte, no solo los gobiernos formulan políticas de desarrollo de proveedores locales, sino que las propias empresas también lo hacen internamente. En ese sentido, las empresas que han promovido el desarrollo de proveedores locales en Colombia, como Ecopetrol, reconocen que dichas iniciativas tienen el potencial de generar externalidades positivas económicas y sociales en las zonas en las que operan (Perry y Palacios, 2013, p. 30).

A pesar de la importancia del desarrollo de proveedores nacionales y locales para el sector extractivo, Colombia no se destaca por contar con un número importante de empresas proveedoras de bienes y servicios al sector extractivo, y la participación de las empresas existentes (en términos de ingresos) en dichos mercados tampoco es significativa, como se explicará a continuación.

En resumen, la posibilidad de que los beneficios económicos de la extracción de recursos naturales no renovables vayan más allá de la extracción misma y puedan irrigarse a la sociedad depende del desarrollo de encadenamientos productivos. Particularmente, si estos involucran actividades de manufactura y tecnología, y si desarrollan capacidad exportadora. En este sentido, alcanzar un mayor grado de integración entre las economías extractivas y las empresas nacionales y locales constituye un reto de política pública para los países que explotan recursos naturales no renovables. Y este documento pretende abordar ese reto mediante el estudio de aquellos factores que afectan la generación de dichos encadenamientos en Colombia.

Ahora bien, no todo es color de rosa en relación con los encadenamientos locales, particularmente aquellos relacionados con la provisión de bienes y servicios a las empresas mineras y de hidrocarburos. Como lo

\footnotetext{
4 Según Perry y Palacios (2013, p. 25) las empresas proveedoras del sector minero-energético que son exportadoras y ofrecen sus bienes y servicios a empresas de otros sectores económicos "son más diversificadas, más exportadoras y más dinámicas".
} 
manifestaron varios funcionarios de empresas extractivas entrevistados, la creación de encadenamientos locales representa oportunidades para las empresas, pero también riesgos. Por una parte, privilegiar la compra nacional o local de bienes y servicios puede encarecer la estructura de costos de las empresas. Asimismo, la dependencia de las empresas extractivas de un reducido grupo de proveedores locales puede incentivar conductas oportunistas de parte estos últimos. Por ejemplo, a través de prácticas anticompetitivas vía acuerdos colusorios (carteles) que aumenten los precios artificialmente ${ }^{5}$, o mediante la coerción política para constreńir la contratación con determinados proveedores locales.

\section{LOS ENCADENAMIENTOS PRODUCTIVOS EN COLOMBIA, EN PERSPECTIVA}

En esta sección se revisan diferentes parámetros que permiten medir el nivel de desarrollo de proveedores locales del sector extractivo en Colombia. Además, algunos de los parámetros presentados se comparan en relación con otros países de la región.
En el sector minero, los encadenamientos hacia atrás más significativos (en términos de valor) consisten en servicios (financieros, transporte y energía) (Reina y Cárdenas, 2008, pp. 36-37). En el caso de la minería metálica, dichos encadenamientos consisten en insumos químicos básicos, metales comunes, servicios financieros, de comercio y transporte (Reina y Cárdenas, 2008, p. 37).

A partir de una matriz insumo-producto Martínez y Aguilar (2013) calcularon que en Colombia el aumento de 1 peso en la producción minera, genera un aumento de 2,44 pesos en la producción bruta nacional ${ }^{6}$. De ese efecto multiplicador de la producción minera, 0,27 pesos se atribuyen al aumento de producción generado por los proveedores directos del sector y 0,26 pesos del aumento de la producción de quienes proveen a los proveedores directos del sector (Martínez y Aguilar, 2013, p. 57). Asimismo, en términos de valor agregado, el "aumento de 1 peso en la producción minera induce un aumento del valor agregado nacional de 1,3 pesos" (Martínez y Aguilar, 2013, p. 57).

El sector minero colombiano presenta un efecto multiplicador similar al de Chile. El

5 Uno de los ingredientes del escándalo que tiene en el ojo del huracán a la empresa estatal petrolera Petrobras en Brasil, consistió en la realización de acuerdos anticompetitivos para amańar licitaciones de proveeduría de la compañía por parte de sus proveedores de servicios y de obras (Leahy, 2015; Segal, 2015). Mientras que las inversiones en contratos de Petrobras superaron los 220 billones de dólares en los últimos años, las pérdidas por casos de corrupción se calculan en 17 billones de dólares en el año 2014 (Leahy, 2015). Con todo, de Oliveira (2015) presenta evidencia empírica que muestra que la corrupción en Petrobras fue mucho mayor en relación con los encadenamientos "aguas abajo" (por ejemplo, que realizan obras para las refinerías) que en relación con los encadenamientos "aguas arriba" (que proveen bienes y servicios para producción de petróleo) y respecto de los cuales aplican las normas sobre contenido local.

$6 \quad$ El multiplicador para minerales metálicos es ligeramente superior: 2,46. 
efecto multiplicador del sector minero chileno, en términos de valor agregado bruto, fue de 1,36 dólares por cada dólar generado por el sector minero en el año 2010 (Comisión Chilena del Cobre, 2013, p. 52). Sin embargo, este multiplicador es inferior al que alcanzaba Chile en el 2013, que la Comisión Chilena del Cobre calculó en 1,66 (p. 52). Por su parte, en el caso peruano, según el Instituto Peruano de Economía (2012, p. 26), el multiplicador de las exportaciones mineras sobre el producto interno bruto es del orden de 1,47 y según Macroconsult (2012, p. 31), el efecto multiplicador en términos de valor agregado es de 2,01.

Por otra parte, volviendo al caso del sector minero colombiano, las cifras presentadas muestran que los encadenamientos en el sector minero son más significativos que los presentes en el sector hidrocarburos, pues el efecto multiplicador de estos en materia de producción es de 1,65 pesos, de los cuales solo 0,16 pesos están asociados a los aumentos de producción de los proveedores directos y 0,09 pesos a los proveedores de proveedores directos (Martínez y Aguilar, 2013, p. 61). Adicionalmente, en términos de aumento de valor agregado, el efecto muiltiplicador es de 1,13 pesos (p. 61) ${ }^{7}$.
A pesar de que las cifras de Martínez y Aguilar (2013) muestran que los encadenamientos mineros han generado mayores multiplicadores productivos -en términos relativos- que los encadenamientos del sector petrolero, Perry y Palacios (2013) señalan que los mercados de bienes y servicios para el sector minero están menos consolidados que los del sector petrolero. En efecto, Perry y Palacios (2013, p. 19) concluyen que en contraste con los proveedores del sector hidrocarburos en Colombia ${ }^{8}$, las empresas proveedoras del sector minero son relativamente pocas, fueron creadas recientemente, presentan menor crecimiento y diversificación y, por lo general, no son exportadoras ni tienen planes de serlo.

Por otra parte, en los encadenamientos "hacia atrás" con el sector extractivo prevalecen las empresas de origen extranjero sobre las nacionales. Según Martínez y Aguilar (2013, p. 78), la demanda anual de bienes y servicios del sector minero e hidrocarburos es de 10 mil millones de dólares, de los cuales $30 \%$ es provisto por nacionales. En el mismo sentido, según Olivera et al. (2011, p. 14), en relación con las empresas prestadoras de servicios petroleros ${ }^{9}$, alrededor del $67 \%$ de los ingresos operacionales (3,2 billones de pesos) corresponden a firmas de origen extranjero y

\footnotetext{
7 El cálculo de Olivera et al. (2011, p. 32) es ligeramente superior al de Martínez y Aguilar (2013, p. 61), pues según los primeros, en términos de valor agregado, el efecto multiplicador de cada peso de valor agregado generado por el sector petrolero es de 1,3 pesos.

8 Los ingresos operacionales de las empresas de servicios petroleros en Colombia en 2011 alcanzaron 12,7 billones de pesos (Perry y Palacios, 2013, p. 9).

9 Aquellas cuyos servicios participan directamente en cualquiera de las etapas de la producción de hidrocarburos.
} 
apenas $33 \%$ de dichos ingresos operacionales (1,6 billones de pesos) a firmas nacionales. En cuanto a nivel de activos, en promedio, la empresa extranjera es tres veces más grande que la colombiana, y las empresas extranjeras tienen el $71 \%$ de los activos (en términos de valor) mientras que las nacionales el $29 \%$ restante (pp. 18-19).

Adicionalmente, si se compara el desarrollo de proveedores locales en el sector de hidrocarburos colombiano (que es principalmente on-shore) con el del sector de hidrocarburos brasilero (que es principalmente off-shore), es evidente el subdesarrollo del primero frente al segundo. Si bien este trabajo no tiene por objeto el realizar comparaciones exhaustivas entre Colombia y otros países, la brecha señalada es proporcional a la activa política industrial del Estado brasilero en esta materia (Ministério de Minas e Energía, 2013; Paulo Sergio Rodrigues Alonso, 2013), que contrasta con los tímidos esfuerzos del Estado colombiano (Martínez y Aguilar, 2013; Perry y Palacios, 2013). Según Olivera et al., "uno de los resultados más evidentes de esta estrategia nacional brasilera ha sido el aumento de la proporción de servicios contratados en el mercado interno, que pasó de $57 \%$ en 2003 a $75 \%$ en el primer semestre de 2009" (2011, p. 55).

Finalmente, la sofisticación de los encadenamientos también es pertinente. En ese sentido, según Cadena y Pinzón (2011, p. 55): "en Colombia, se tienen compras de alta frecuencia con bajo nivel de sofisticación, por lo que se esperaría lograr una producción con mayor nivel, dirigida al mercado nacional e internacional".
En resumen, las cifras sobre proveedores locales del sector extractivo colombiano muestran que el nivel de desarrollo no es significativo en términos de participación en los ingresos, en tamaño de las empresas, en capacidad exportadora y en nivel de innovación. Hay más "espacio" para profundizar los encadenamientos productivos (hacia atrás) entre el sector extractivo y el resto de la economía, y el Estado colombiano podría jugar un rol importante en este sentido.

\section{CUELLOS DE BOTELLA Y FACTORES DE ÉXITO}

En esta sección se presentan los principales cuellos de botella identificados en el desarrollo de proveedores locales y nacionales para el sector extractivo en Colombia. Si bien las estructuras de costos y tipo de bienes y servicios demandados por el sector minero y el sector hidrocarburos son diferentes (anotación que también aplica para las empresas mineras que explotan diferentes tipos de minerales), se observan similitudes importantes en las dificultades para desarrollar proveedores locales. Se advierte que si bien el objetivo principal de esta sección es la identificación de cuellos de botella, se estudian también los factores de éxito que determinan el liderazgo de un proveedor de bienes o servicios. Estos factores de éxito sirven de "espejo inverso" para evaluar los cuellos de botella para la creación y el desarrollo de proveedores competitivos, con capacidad para innovar y exportar.

Antes de entrar en materia, vale la pena aclarar que el surgimiento de encadenamien- 
tos, particularmente de clusters, depende de diversos factores que los favorecen (o los disuaden) y que la literatura ha divido en tres categorías: i) prerrequisitos (dotación de recursos inicial), ii) eventos activadores (triggers) y iii) procesos que se "autoalimenten" (spillovers) (Cadena y Pinzón 2011). Este trabajo se enfocará en el primer tipo de categoría, los prerrequisitos, que pueden incluir, por ejemplo, la existencia de mano de obra calificada, de asociaciones gremiales, adecuada infraestructura, etc. No obstante lo anterior, se reconoce que el surgimiento de encadenamientos productivos no solo depende de estos factores favorables que anteceden a la explotación, sino también de políticas específicas diseñadas e implementadas por el Gobierno, así como empresas líderes en la materia que tengan influencia sobre el resto del sector, y que haya espacios de interacción entre los diferentes actores involucrados para que puedan trabajar conjuntamente en el desarrollo de encadenamientos productivos.

A diferencia de lo que ocurre con otro tipo de actividades económicas que presentan flexibilidad en cuanto a las zonas en las cuales desarrollan sus operaciones, las empresas del sector extractivo dependen del lugar en el cual se encuentra ubicado su yacimiento. Por eso, este tipo de recursos son denominados en Inglés point-source natural resources. Precisamente, por la ubicación de los yacimientos, las compañías del sector minero y petrolero operan en lugares apartados y con bajos niveles de desarrollo (Martínez y Aguilar, 2013; Perry y Palacios, 2013). Los contextos en los cuales operan estos proyectos, por lo general, se caracterizan por bajos niveles de capital humano e institucional que dificultan el desarrollo de cualquier emprendimiento, no solo aquellos relacionados con el sector extractivo.

Según los resultados de una encuesta realizada a las empresas vinculadas al sector de la Minería de Gran Escala (Martínez y Aguilar 2013, p. 78), los desafíos de los proveedores locales del sector son los siguientes:

- Tiempos de entrega ${ }^{10}$.

- Baja disponibilidad de equipos.

- Escasez de mano de obra.

- Pocas empresas que tienen capacidad para participar (número de empresas no son suficientes para cubrir demanda nacional).

- Bajo cumplimiento de estándares de producción o servicios.

- Problemas con calidad de bienes o servicios prestados.

- No existe registro centralizado de proveedores locales y regionales que permita identificar oferta (sin incurrir en grandes costos de transacción).

- El servicio de transporte es limitado.

Otros estudios han identificado dos retos adicionales que no "salieron a flote" en la encuesta citada: las dificultades para innovar y para exportar. Según Perry y Palacios (2013,

10 En el caso de Brasil, este es uno de los costos más altos que el sector petrolero ha tenido que asumir por cuenta de los proveedores locales (de Oliveira, 2015). 
p. 25), parece que existe una relación directa entre el tamaño de la empresa y su experiencia (tiempo de operación) con su crecimiento, diversificación y capacidad para exportar. Como se explicó al comienzo de este documento, los proveedores nacionales del sector extractivo, no se caracterizan por su gran tamańo ni por su experiencia en el sector (en comparación los proveedores internacionales). Las mismas empresas proveedoras identifican su dificultad para diversificar y exportar y lo atribuyen, entre otros, a factores externos. Según Perry y Palacios (2013, p. 22), las empresas proveedoras consideran que la política pública no ayuda a exportar (apreciación, infraestructura, falta de programas de apoyo) y que el acceso a la financiación no es adecuado.

Por otra parte, a través de encuestas y entrevistas a grupos focalizados, Castro et al. (2012) identificaron algunas dificultades que se han presentado en el sector petrolero para desarrollar proveedores:

- La falta de acceso a certificaciones de calidad de proveedores (p. ej., IsO, OHSAS, HACCP e Icontec) (Castro et al., 2012, p. 78).

- Los obstáculos financieros, logísticos y tecnológicos de los proveedores de bienes y servicios del sector petrolero les impi- den ser competitivos fuera de sus regiones (Castro et al., 2012, p. 79).

- Las empresas proveedoras del sector petrolero carecen de cultura asociativa que les permita realizar proyectos conjuntos para aumentar la productividad o lograr economías de escala ${ }^{11}$ (Castro et al., 2012, p. 80).

- La oferta educativa en las regiones en las cuales están ubicados los proyectos no cubre la demanda requerida por el sector. A pesar de los esfuerzos gubernamentales (a través del SENA), los programas educativos no se enfocan en desarrollar competencias específicas para el sector petrolero (Castro et al., 2012, p. 82). Asimismo, muchas de esas regiones carecen de universidades que puedan ofrecer educación profesional, y si tales instituciones existen, no cuentan con programas específicos para el sector (p. 83).

- Otro problema asociado es la falta de experiencia de los profesionales de estas regiones en el sector petrolero ${ }^{12}$.

- Baja capacidad de innovación de proveedores de sector petrolero (Castro et al., 2012, p. 85).

Por otra parte, en las encuestas realizadas por ANDI (2009, p. 39) para identificar los fac-

\footnotetext{
11 En contraste, en los mercados internos de tamaño considerable, como en Australia, influye positivamente en el desarrollo de proveedores locales la escala de la demanda interna del sector minero (Martínez y Aguilar, 2013).

13 Según Castro et al. (2012, p. 83): "Este vacío en la formación, ha llevado a las operadoras a seleccionar profesionales del personal de las empresas contratistas. Esta situación, sumada a la diferencia salarial entre operadores y contratistas, ha generado una alta circulación de empleados, dificultando procesos de gestión del conocimiento y de continuidad en los procesos operativos de los contratistas. Adicionalmente, el fenómeno reduce el ritmo de desarrollo del empresariado local”.
} 
tores que explican el "liderazgo competitivo" de las empresas líderes en provisión de bienes y servicios al sector petrolero, tres factores fueron resaltados como determinantes del éxito ${ }^{13}$ :

- Conocimiento y experiencia del sector de hidrocarburos.

- Calidad de los bienes y servicios.

- Cumplimiento de estándares.

Estos resultados, que enfatizan en la calidad de los bienes y servicios para los respectivos sectores como principales retos de proveedores locales, coinciden con lo expresado por las empresas del sector extractivo para la realización de este trabajo.

Volviendo a los resultados de la encuesta de ANDI (2009), también fueron identificados como factores explicativos del éxito de los proveedores líderes ${ }^{14}$, los siguientes:

- Uso de tecnología de punta.

- Oportunidad de entrega.

- Prestigio internacional.

- Capacidad de innovación.

- Acceso a financiamiento.
Adicionalmente, vale la pena resaltar de la encuesta de ANDI (2009) que menos del $40 \%$ de los encuestados consideraron los bajos costos como característica de los proveedores líderes. Este hallazgo va en contravía de la intuición según la cual la baja penetración de los proveedores locales está principalmente ligada la constante búsqueda de reducción de la estructura de costos de las áreas de compras $\mathrm{y}$ abastecimiento de las empresas mineras y petroleras.

No obstante lo anterior, las empresas de los sectores mineros y petroleros han tenido que ajustar su estructura de costos debido a la fuerte reducción de los precios de los commodities en los últimos ańos ${ }^{15}$. El deterioro de los precios mundiales de estos bienes ha tenido un impacto fuerte en las finanzas de las empresas. En consecuencia, algunas compañías han optado por cerrar o suspender proyectos ${ }^{16} \mathrm{y}$ por disminuir su ritmo de gastos e inversiones ${ }^{17}$, lo cual ha impactado también la contratación de proveedores nacionales y locales. En diferentes municipios de los Llanos Orientales se manifiesta el ocaso de las empresas que proveen bienes y servicios a las petroleras por cuenta de

13 Más del $85 \%$ de los encuestados identificaron estos factores como factores de éxito.

14 Entre el 55 y el $70 \%$ de los encuestados identificaron estos factores como factores de éxito.

15 Los minerales metálicos y el carbón fueron los primeros commodities cuyos precios disminuyeron. El níquel bajó vertiginosamente desde mediados de 2007, se recuperó a mediados de 2009 y ha bajado de precio desde comienzos de 2011 hasta la fecha. El precio del oro y del carbón se ha disminuido sostenidamente desde finales de 2012. Finalmente, los precios del petróleo pasaron de casi 115 dólares por barril en junio de 2014 a menos de 50 dólares por barril actualmente (Raval y David, 2015).

16 Por ejemplo, la decisión de una empresa filial de Ecopetrol, a comienzos de 2015, de cerrar siete pozos en Casanare, cuya explotación no era rentable por la caída de los precios (Vega, 2015).

17 Ver Lafuente (2015). No solo Ecopetrol anunció recortes en inversión y gastos, también empresas privadas como Pacific Rubiales y Canacol Energy lo han anunciado (Moreno, 2015). 
la caída de los precios. Este es el caso de Puerto Gaitán, Meta, en donde hoteleros, mecánicos $\mathrm{y}$ transportadoras prácticamente ya no tienen trabajo (Güesguán, 2015; Lafuente, 2015).

Quizás en las encuestas arriba mencionadas este último aspecto no aparecía mencionado puesto que las mismas se realizaron cuando los precios de los commodities todavía eran muy favorables. No obstante lo anterior, la volatilidad de los precios de los recursos naturales no renovables es un factor que dificulta la consolidación de proveedores nacionales y locales. Particularmente, en la medida en que muchos de los proveedores incipientes dependen de una sola empresa extractiva (su único cliente) o, en el mejor de los casos, dependen de varias empresas del sector extractivo. Son muy pocos los proveedores que tienen la capacidad de trascender del sector extractivo para atender otros mercados, como ha ocurrido en otros países como Australia, Canadá, Noruega y Suecia. Además, el bajo acceso al mercado financiero que se mencionó arriba, aunado a la fragilidad financiera de las empresas proveedoras, hace casi imposible que estas puedan cubrirse de la volatilidad de los mercados.

Por último, también es pertinente considerar de qué manera el Estado y las empresas del sector extractivo han promovido el desarrollo de proveedores nacionales y locales. Una parte de la explicación para las dificultades de las empresas locales consiste en que el Estado no presta bienes públicos básicos (por ejemplo, infraestructura de transporte) y tampoco tiene como objetivo claro (y priorizado) el desarrollo de este sector. En efecto, la reciente literatura que ha evaluado la política implementada por el actual Gobierno en relación con la promoción de encadenamientos productivos con el sector extractivo muestra que, en contraste con diferentes instrumentos y programas puestos en marcha en países como Brasil, Canadá y Chile, en Colombia estos esfuerzos estatales son apenas incipientes y aparentemente con pobres resultados ${ }^{18}$.

La otra parte de la explicación para las dificultades de las empresas locales también tiene que ver con la falta de interés de la mayoría de las empresas del sector extractivo por implementar políticas de compras de bienes y servicios locales más allá de programas de responsabilidad social. Quizás la única empresa del sector extractivo que ha asumido esta tarea como una que está en el "núcleo" de su negocio es Ecopetrol. Desde el año 2006, Ecopetrol implementó el Programa de Desarrollo

18 El único programa de desarrollo de proveedores locales para el sector minero-energético, a nivel nacional, de origen estatal, es el capítulo "Hidrocarburos y Minería” del Programa de Innovación Abierta (innpulsa Colombia) de Bancoldex. El programa busca acercar a empresas minero-energéticas con proveedores locales para la solución de problemas específicos que requieren soluciones técnicas. Sin embargo, en el primer programa piloto de inNpulsa solo participó una empresa minera, Cementos Argos, las demás están relacionadas con el sector hidrocarburos. Aún no existe ninguna evaluación del desarrollo de este programa piloto ni de los resultados que puede haber producido. Pueden existir otras herramientas o programas de origen estatal que son accesibles para las empresas que pretendan ofrecer bienes y servicios al sector minero-energético, pero que no están diseñadas particularmente para promover proveedores locales en dicho sector (Perry y Palacios, 2013). 
de Proveedores y es quizás la empresa que ha hecho el mayor esfuerzo por desarrollar este tipo de programas. El objeto del mismo sería "reversar algunos efectos nocivos de la política de centralización de las compras establecida por la compañía en 2004 sobre proveedores locales" (Perry y Palacios, 2013, p. 30). El programa busca incentivar la formalización y certificación de los proveedores. No obstante lo anterior, incluso Ecopetrol, bajo los nuevos precios del petróleo, está revaluando aquellas actividades que se salen de su "core" de negocios, según informó una de las personas entrevistadas.

En el sector minero, el Cerrejón cuenta con un programa de desarrollo de proveedores llamado "Programa de fabricación nacional de partes" orientado, como su nombre lo indica, a "reparación de maquinaria y equipo y suministro de repuestos" (Perry y Palacios 2013, p. 32). No obstante la misma empresa reconoce que el programa no arrojó los resultados esperados. Actualmente, menos del $1 \%$ de los bienes comprados y cerca del $10 \%$ de los servicios contratados por la empresa corresponden a proveedores de La Guajira. Por su parte, la empresa Cerromatoso creó una Red de 20 proveedores locales en Córdoba, que representan alrededor del $10 \%$ de las compras nacionales (Perry y Palacios 2013, p. 32). Además, desde mediados de 2014, Cerromatoso ha ejecutado diferentes estrategias para identificar potenciales proveedores regionales.

Para resumir lo presentado en esta sección, a continuación procederé a clasificar los diferentes factores que inciden en el éxito del desarrollo de proveedores locales agrupándolas por categorías. Esto permitirá asignar roles y responsabilidades en el capítulo final, de implicaciones de políticas (tabla 1).

\section{TABLA 1. CATEGORÍAS DE FACTORES DE ÉXITO} EN EL DESARROLLO DE PROVEEDORES

\begin{tabular}{|c|c|}
\hline Categoría & Subcategoría \\
\hline 1. Calidad & $\begin{array}{l}\text { 1.1 Tiempos de entrega. } \\
\text { 1.2 Mano de obra calificada. } \\
\text { 1.3 Uso de tecnología adecuada. } \\
\text { 1.4 Obtención de certificados de } \\
\text { calidad para acreditar procesos. }\end{array}$ \\
\hline 2. Financiación & $\begin{array}{l}2.1 \text { Acceso a financiación. } \\
\text { 2.2 Contar con colaterales idó- } \\
\text { neos. }\end{array}$ \\
\hline 3. Infraestructura & $\begin{array}{l}\text { 3.1 De transporte desde el inte- } \\
\text { rior del país hacia los puertos } \\
\text { o destinos finales. }\end{array}$ \\
\hline 4. Información & $\begin{array}{l}\text { 4.1 Acceso a información sobre } \\
\text { existencia de proveedores } \\
\text { nacionales / locales. } \\
\text { 4.2 Acceso a información sobre } \\
\text { estándares requeridos por } \\
\text { el sector extractivo y sobre } \\
\text { necesidades. }\end{array}$ \\
\hline 5. Asociatividad & $\begin{array}{l}\text { 5.1 Capacidad para asociarse con } \\
\text { empresas proveedores para } \\
\text { obtener mayor escala. } \\
\text { 5.2 Capacidad para asociarse con } \\
\text { empresas proveedores para } \\
\text { innovar. }\end{array}$ \\
\hline $\begin{array}{l}\text { 6. Volatilidad de } \\
\text { los precios de } \\
\text { los commodi- } \\
\text { ties }\end{array}$ & $\begin{array}{l}\text { 6.1 No dependencia del pro- } \\
\text { veedor respecto de una sola } \\
\text { empresa extractiva. } \\
\text { 6.2 Ampliar portafolio de produc- } \\
\text { tos o servicios a otros sectores } \\
\text { diferentes de la minería y los } \\
\text { hidrocarburos. }\end{array}$ \\
\hline $\begin{array}{l}\text { 7. Política públi- } \\
\text { ca para desa- } \\
\text { rrollo de enca- } \\
\text { denamientos } \\
\text { productivos }\end{array}$ & $\begin{array}{l}\text { 7.1 Programas y herramientas im- } \\
\text { pulsadas por el Estado junto } \\
\text { con sector extractivo (zanaho- } \\
\text { ria). } \\
\text { 7.2 Hacer efectiva la legislación } \\
\text { que le pide a concesionarios } \\
\text { mineros y petroleros que } \\
\text { contraten bienes y servicios } \\
\text { nacionales y locales (garrote). }\end{array}$ \\
\hline
\end{tabular}




\section{IMPLICACIONES DE POLÍTICA PÚBLICA}

El presente documento comenzó explicando la pertinencia del tema escogido, particularmente por el impacto económico de los encadenamientos productivos de la economía con el sector extractivo, y luego presentó el estado de dichos encadenamientos (hacia atrás o upstream) en Colombia. Posteriormente, describió los principales factores que la literatura existente ha identificado como incluyentes para el éxito del desarrollo de los encadenamientos entre empresas nacionales y empresas mineras y de hidrocarburos. Estos factores se clasificaron en siete grandes categorías: i) calidad, ii) financiación, iii) infraestructura, iv) información, v) asociatividad, vi) volatilidad de los precios de los commodities y vii) política pública para desarrollo de encadenamientos productivos.

El objetivo de este documento, además de identificar los factores arriba enunciados, consiste en derivar implicaciones de política para el sector extractivo. La lectura tradicional sobre el sector extractivo, es que la explotación de los recursos naturales generalmente tiene lugar en modelos de "enclave", sin muchos encadenamientos con sectores industriales y que esto ocurra sin que sea necesario utilizar mucha mano de obra por ser intensiva en capital (Humphreys, Sachs y Stiglitz, 2007, p. 4). En ese mismo sentido, Gelb (2011) argumenta que en el caso de los exportadores de minerales, los encadenamientos productivos entre estos y el resto de la economía son "relativamente limitados".

Sin embargo, Wright y Czelusta (2002), Herzer y Nowak-Lehmann (2004) y Blom- ström y Kokko (2007), cuestionan el supuesto trade-offentre el desarrollo a partir de commodities y a partir de la industria, generalmente planteado bajo el marco de la teoría de la maldición de los recursos naturales. Quizás bajo ese contexto fue concebido el trabajo investigativo realizado por las facultades de Administración y de Ingeniería de la Universidad de los Andes, junto con el Departamento Nacional de Planeación (DNP) de Colombia, que buscó "identificar potencialidades para la creación de conglomerados de negocios y la consolidación de ramas industriales, como producto del aumento de la actividad (base reservas y producción) petrolera, gasífera y carbonera en Colombia" (Cadena y Pinzón, 2011, pp. 419-50).

Precisamente, el desarrollo de industrias exportadoras y de alta tecnología alrededor del sector extractivo, como lo ha hecho Noruega, muestra que más allá de la teoría podría haber condiciones propicias para que el sector extractivo no genere externalidades negativas que impidan el desarrollo del sector industrial, sino que puedan generar círculos virtuosos de crecimiento. Por supuesto, hay un camino largo entre las instituciones de Noruega y de Colombia, lo cual puede explicar por qué el primero, desde los años setenta, ha logrado construir tanto a partir de su sector extractivo, mientras que el segundo, que empezó a extraer petróleo cuarenta años antes, apenas está dando los primeros pasos para generar encadenamientos productivos con empresas de "clase mundial", es decir empresas proveedoras competitivas a nivel internacional.

Volviendo al tema central de este trabajo, a continuación se presentarán recomendacio- 
nes sobre cómo superar las dificultades que fueron clasificadas en seis categorías identificando el rol que tendría el sector estatal, el sector extractivo y las empresas proveedoras (tabla 2). Como se verá a continuación, en la medida en que se asignan roles a diferentes actores, incluyendo no estatales, superar los retos requiere mucho más que la formulación de políticas públicas: se requiere un trabajo estrecho entre agentes del sector privado (sector extractivo, gremios, proveedores) y entre el sector privado y el Estado como ocurre en Brasil, Chile y Australia.

TABLA 2. RECOMENDACIONES PARA SUPERAR CUELLOS DE BOTELLA

\begin{tabular}{|c|c|c|c|}
\hline Factor & Sector extractivo & Estado & Empresa proveedora \\
\hline \multicolumn{4}{|c|}{ 1. Calidad } \\
\hline $\begin{array}{l}\text { 1.1 Tiempos de } \\
\text { entrega }\end{array}$ & $\begin{array}{l}\text { - Trabajar de la mano de } \\
\text { los proveedores actuales } \\
\text { y capacitar a proveedores } \\
\text { potenciales en mejores } \\
\text { prácticas en procesos de } \\
\text { producción. }\end{array}$ & $\begin{array}{l}\text { - Identificar, estructurar y eje- } \\
\text { cutar obras de infraestructu- } \\
\text { ra de transporte críticas para } \\
\text { el sector extractivo. }\end{array}$ & $\begin{array}{l}\text { - Invertir en mejorar procesos. } \\
\text { Lo anterior puede realizarse } \\
\text { de la mano con la empresa } \\
\text { extractiva. Por ejemplo, en } \\
\text { Brasil Petrobras estableció } \\
\text { programas de monitoreo } \\
\text { detallado a sus proveedores } \\
\text { estratégicos para trabajar } \\
\text { estrechamente en encon- } \\
\text { trar soluciones (de Oliveira, } \\
\text { 2015). }\end{array}$ \\
\hline $\begin{array}{l}\text { 1.2 Mano de obra } \\
\text { calificada }\end{array}$ & \multicolumn{2}{|c|}{$\begin{array}{l}\text { - En convenio con sector extractivo, organizar a través del SE- } \\
\text { NA y de universidades públicas y privadas programas especí- } \\
\text { ficamente orientados a cubrir demanda laboral del sector }{ }^{19} \text {. }\end{array}$} & $\begin{array}{l}\text { - Financiar capacitación de } \\
\text { sus actuales trabajadores. }\end{array}$ \\
\hline $\begin{array}{l}1.3 \text { Uso de tecnolo- } \\
\text { gía adecuada }\end{array}$ & $\begin{array}{l}\text { - Informar y capacitar a los } \\
\text { proveedores en las tecno- } \\
\text { logías que se exigen como } \\
\text { parte de sus estándares de } \\
\text { calidad para la producción } \\
\text { de bienes y prestación de } \\
\text { servicios. }\end{array}$ & $\begin{array}{l}\text { - Otorgar línea de crédito es- } \\
\text { pecial a través de Bancoldex } \\
\text { para que empresas pro- } \\
\text { veedoras puedan adquirir } \\
\text { tecnología adecuada. }\end{array}$ & - Adquisición de tecnología \\
\hline
\end{tabular}

19 Un caso para revisar en materia de calificación de profesionales es el de Brasil. Entre 2006-2011 se realizaron inversiones de 292 millones de reales utilizados en la capacitación de alrededor de 97.000 profesionales en 17 estados, en 185 categorías ocupacionales (desde el nivel básico hasta el superior). A la fecha, han capacitado más de 200.000 trabajadores (de Oliveira, 2015, p. 20). Para los estudiantes que no tenían vinculación con el sector extractivo, la capacitación fue financiada al 100 \% por el "Programa de Movilización Nacional de la Industria de Petróleo y Gas" (Prominp). Para estudiantes vinculados con empresas del sector extractivo, Prominp financia el $50 \%$ de la capacitación y el resto las empresas (Rodrigues Alonso, 2013). 


\begin{tabular}{|c|c|c|c|}
\hline Factor & Sector extractivo & Estado & Empresa proveedora \\
\hline $\begin{array}{l}\text { 1.4 Obtención de } \\
\text { certificados de } \\
\text { calidad para } \\
\text { acreditar pro- } \\
\text { cesos }\end{array}$ & \multicolumn{3}{|c|}{$\begin{array}{l}\text { - Realizar un diagnóstico nacional de oferta y demanda, identificar cuellos de botella y realizar } \\
\text { talleres con proveedores para que interactúen con la industria para mejorar la competitividad } \\
\text { de los primeros. Por ejemplo, este tipo de actividades han sido desarrolladas en Brasil bajo el } \\
\text { esquema de Prominp (Rodrigues Alonso, 2013). } \\
\text { - Adicionalmente, las empresas del sector extractivo (como lo ha hecho Ecopetrol) pueden } \\
\text { realizar convenios con universidades y otras organizaciones para implementar sistemas de } \\
\text { gestión y fortalecer proveedores. }\end{array}$} \\
\hline \multicolumn{4}{|c|}{ 2. Financiación } \\
\hline $\begin{array}{l}2.1 \text { Acceso a finan- } \\
\text { ciación }\end{array}$ & \multicolumn{2}{|c|}{$\begin{array}{l}\text { - El Estado, junto con el sector financiero, puede promover } \\
\text { nuevos canales de financiación para adquisición de tecnolo- } \\
\text { gía por parte de proveedores. Además, en la medida en que } \\
\text { el trabajo con las empresas extractivas y los proveedores sea } \\
\text { más estrecho, los contratos entre estas y aquellas pueden } \\
\text { servir de colateral para la financiación. } \\
\text { Un buen ejemplo de la anterior iniciativa, consiste en el plan } \\
\text { de financiamiento operado por el Programa Progredir en } \\
\text { Brasil, con participación de diez bancos. Se trata de un pro- } \\
\text { grama conjunto de Petrobras y Prominp, que fue lanzado en } \\
\text { el año } 2011 \text {. A la fecha ha otorgado } 7 \text { billones de reales (7,8 } \\
\text { mil millones de dólares) en créditos a } 570 \text { proveedores en } 21 \\
\text { Estados de Brasil. Los créditos otorgados a los proveedores } \\
\text { están garantizados por los contratos suscritos entre estos y } \\
\text { Petrobras (Rodrigues Alonso 2013). }\end{array}$} & \\
\hline \multicolumn{4}{|c|}{ 3. Infraestructura } \\
\hline $\begin{array}{l}\text { 3.1 De transporte } \\
\text { al interior del } \\
\text { país }\end{array}$ & $\begin{array}{l}\text { - Promover la realización de } \\
\text { proyectos público-privados } \\
\text { para la construcción de } \\
\text { infraestructura. }\end{array}$ & $\begin{array}{l}\text { - Identificar, estructurar y eje- } \\
\text { cutar obras de infraestructu- } \\
\text { ra de transporte críticas para } \\
\text { el sector extractivo. }\end{array}$ & \\
\hline \multicolumn{4}{|c|}{ 4. Información } \\
\hline $\begin{array}{l}\text { 4.1 Acceso a infor- } \\
\text { mación sobre } \\
\text { proveedores }\end{array}$ & \multicolumn{2}{|c|}{$\begin{array}{l}\text { - En convenio con Cámaras de Comercio, asociaciones gre- } \\
\text { miales, autoridades locales y empresas del sector extractivo } \\
\text { generar Observatorios y Registros para identificar oferta } \\
\text { existente en economías locales (Cadena y Pinzón, 2011). } \\
\text { - Adicionalmente, en convenio con Cámaras de Comercio, } \\
\text { asociaciones gremiales, autoridades locales y empresas del } \\
\text { sector extractivo se podrían organizar "ferias inversas" en las } \\
\text { cuales las empresas extractivas expongan sus necesidades } \\
\text { puntuales, para que la demanda y la oferta de soluciones } \\
\text { puedan encontrarse. }\end{array}$} & $\begin{array}{l}\text { - Participar activamente en } \\
\text { las actividades organizadas } \\
\text { por empresas del sector } \\
\text { extractivo, agremiaciones } \\
\text { y Gobierno en materia de } \\
\text { proveeduría de bienes y } \\
\text { servicios al sector extractivo. }\end{array}$ \\
\hline $\begin{array}{l}\text { 4.2 Acceso a infor- } \\
\text { mación sobre } \\
\text { estándares y } \\
\text { sobre necesi- } \\
\text { dades }\end{array}$ & $\begin{array}{l}\text { - Publicar las políticas de } \\
\text { compra a proveedores, las } \\
\text { marcas aceptadas (como lo } \\
\text { hace Ecopetrol), los estánda- } \\
\text { res tanto en procesos como } \\
\text { los productos y servicios }\end{array}$ & $\begin{array}{l}\text { - Realizar estudios de viabi- } \\
\text { lidad de oportunidades de } \\
\text { negocio para proveedores } \\
\text { nacionales. En Brasil, por } \\
\text { ejemplo, la Agencia Nacio- } \\
\text { nal de Petróleo, Gas y }\end{array}$ & $\begin{array}{l}\text { - Participar activamente en } \\
\text { las actividades organizadas } \\
\text { por empresas del sector } \\
\text { extractivo, agremiaciones y } \\
\text { Gobierno en materia de }\end{array}$ \\
\hline
\end{tabular}




\begin{tabular}{|c|c|c|c|}
\hline Factor & Sector extractivo & Estado & Empresa proveedora \\
\hline & $\begin{array}{l}\text { demandados. Además, } \\
\text { capacitar a proveedores } \\
\text { actuales y prospectivos, } \\
\text { generar espacios periódicos } \\
\text { para revisarlo (como lo hace } \\
\text { Ecopetrol). }\end{array}$ & $\begin{array}{l}\text { Energías Renovables contra- } \\
\text { tó una universidad para que } \\
\text { elaborara un estudio de las } \\
\text { condiciones necesarias para } \\
\text { incrementar la participación } \\
\text { de proveedores nacionales } \\
\text { en el sector de hidrocarbu- } \\
\text { ros (de Oliveira 2015). }\end{array}$ & $\begin{array}{l}\text { proveeduría de bienes y } \\
\text { servicios al sector extractivo }\end{array}$ \\
\hline \multicolumn{4}{|c|}{ 5. Capacidad de asociación } \\
\hline $\begin{array}{l}\text { 5.1 Capacidad para } \\
\text { asociarse con } \\
\text { empresas pro- } \\
\text { veedores para } \\
\text { obtener mayor } \\
\text { escala }\end{array}$ & $\begin{array}{l}\text { - Las empresas del sector ex- } \\
\text { tractivo (como lo ha hecho } \\
\text { Ecopetrol) pueden ofrecer } \\
\text { capacitación a proveedores } \\
\text { en gerencia de proyectos y } \\
\text { en planeación. }\end{array}$ & & $\begin{array}{l}\text { - A través de las asociaciones } \\
\text { gremiales podría estrechar- } \\
\text { se el trabajo entre provee- } \\
\text { dores y propiciar alianzas } \\
\text { estratégicas para proyectos } \\
\text { concretos que empresas del } \\
\text { sector extractivo les encar- } \\
\text { guen. }\end{array}$ \\
\hline $\begin{array}{l}5.2 \text { Capacidad para } \\
\text { asociarse con } \\
\text { empresas pro- } \\
\text { veedores para } \\
\text { innovar }\end{array}$ & $\begin{array}{l}\text { - La generación de innova- } \\
\text { ción abierta, como la que } \\
\text { actualmente promueve } \\
\text { el programa innpulsa de } \\
\text { Bancoldex ha dado resulta- } \\
\text { dos en Chile } 20 \text {. Sin embargo, } \\
\text { requiere un mayor compro- } \\
\text { miso en recursos y continui- } \\
\text { dad. }\end{array}$ & \multicolumn{2}{|c|}{$\begin{array}{l}\text { - Dentro de las actividades del Observatorio arriba menciona- } \\
\text { do, también puede incluirse realizar estudios de inteligen- } \\
\text { cia de mercado que orienten a los diferentes actores sobre } \\
\text { cuáles son las oportunidades más viables para invertir en } \\
\text { generar innovación (Cadena y Pinzón, 2011). }\end{array}$} \\
\hline \multicolumn{4}{|c|}{ 6. Volatilidad de los precios de los commodities } \\
\hline $\begin{array}{l}6.1 \text { Incentivos para } \\
\text { diversificar }\end{array}$ & $\begin{array}{l}\text { - Dentro de los programas de } \\
\text { desarrollo de proveedores } \\
\text { locales o nacionales, las } \\
\text { empresas extractivas deben } \\
\text { incentivar que sus provee- } \\
\text { dores desarrollen clientes } \\
\text { por fuera de su región y en } \\
\text { otros sectores económicos. }\end{array}$ & $\begin{array}{l}\text { - Bajo un contexto de precios } \\
\text { bajos, muchas de las em- } \\
\text { presas proveedoras serán } \\
\text { inviables y el Estado puede } \\
\text { promover la "reconversión" } \\
\text { de algunas de las empresas } \\
\text { hacia otras actividades eco- } \\
\text { nómicas. }\end{array}$ & $\begin{array}{l}\text { - Identificar oportunidades de } \\
\text { diversificación (de produc- } \\
\text { tos y geográficas) desde el } \\
\text { comienzo de su actividad } \\
\text { económica. }\end{array}$ \\
\hline
\end{tabular}

20 Desde el año 2008, una alianza entre el sector público, el sector minero (BHP Billiton) y organizaciones privadas enfocadas en innovación y emprendimiento lanzó un programa de "Desarrollo de Proveedores de Clase Mundial" (Martínez y Aguilar, 2013). El formato del programa es similar al impulsado en Colombia a través de inNpulsa Colombia, en el cual las empresas mineras identifican retos, los proveedores potenciales presentan propuestas para resolver dichos retos y luego se escoge la mejor propuesta para desarrollar una solución técnica de manera conjunta entre la empresa y el proveedor. 


\begin{tabular}{|c|c|c|c|}
\hline Factor & Sector extractivo & Estado & Empresa proveedora \\
\hline \multicolumn{4}{|c|}{ 7. Política pública } \\
\hline $\begin{array}{l}\text { 7.1 Programas y } \\
\text { herramientas } \\
\text { impulsadas por } \\
\text { el Estado junto } \\
\text { con el sector } \\
\text { extractivo }\end{array}$ & \multicolumn{3}{|c|}{$\begin{array}{l}\text { - Estructurar e implementar de manera conjunta un “Programa nacional de proveedores y ser- } \\
\text { vicios” para el sector extractivo (Cadena y Pinzón, 2011). } \\
\text { Quizás el programa más interesante para tener en cuenta en la región es el “Programa de Mo- } \\
\text { vilización Nacional de la Industria de Petróleo y Gas” (Prominp), creado en el } 2003 \text { en virtud } \\
\text { del Decreto } 4925 \text { con la coordinación del Ministerio de Minas y Energía de Brasil²1, para au- } \\
\text { mentar la participación de compañías brasileras en la prestación de servicios de proyectos de } \\
\text { petróleo y gas (Olivera et al., 2011)22. Para tal efecto, pretende reunir a los diferentes agentes } \\
\text { de la industria (Gobierno, operadores, asociaciones gremiales, representantes de industria, } \\
\text { bancos de desarrollo, etc.) con el fin de "maximizar el contenido local a partir de bases com- } \\
\text { petitivas y sostenibles” (Ministério de Minas y Energía de Brasil, 2013). Hay un trabajo estrecho } \\
\text { entre empresas del sector y asociaciones gremiales y los programas de Prominp están alinea- } \\
\text { dos con la Política de Contenido Local de Petrobras (Rodrigues Alonso, 2013). }\end{array}$} \\
\hline $\begin{array}{l}7.2 \text { Hacer efectiva } \\
\text { la legislación } \\
\text { sobre adquisi- } \\
\text { ción nacional }\end{array}$ & $\begin{array}{l}\text { - Tanto la legislación min } \\
\text { lombia, así como los co } \\
\text { templan artículos y cláu } \\
\text { concesionarios que pro } \\
\text { cios a la industria nacio } \\
\text { en pie de igualdad en c } \\
\text { bles en el mercado naci } \\
\text { Estado-empresa debe s } \\
\text { la pena que el Estado cc } \\
\text { sector extractivo sobre } \\
\text { cos de dichas normas. } \\
\text { Por ejemplo, el program } \\
\text { exigencias que hacen la } \\
\text { bierno de Brasil en relac } \\
\text { y servicios nacionales. P } \\
\text { medición de contenido }\end{array}$ & $\begin{array}{l}\text { drocarburos de Co- } \\
\text { ión suscritos, con- } \\
\text { s se exhorta a los } \\
\text { irir bienes y servi- } \\
\text { estos se encuentren } \\
\text { ente a los disponi- } \\
\text { eal en la relación } \\
\text { or incentivos, vale } \\
\text { una señal creíble al } \\
\text { e en términos jurídi- } \\
\text { asil se apoya en las } \\
\text { orgadas por el Go- } \\
\text { mínimas de bienes } \\
\text { on un modelo de }\end{array}$ & \\
\hline
\end{tabular}

Para finalizar, vale la pena reiterar dos puntos afirmados en el cuerpo del documento. En primer lugar, que hay espacio para que desde la academia se profundice aún más en los particulares cuellos de botella que aquejan los diferentes sectores mineros y que quizás

21 El Comité Directivo de Prominp está presidido por el Ministro de Minas y Energía e incluye los siguientes miembros: el presidente de Petrobras, el presidente del Banco de Desarrollo (BNDEs), el presidente del Instituto Brasileiro do Petróleo, Gás e Biocombustíveis (IBP) y el director general de la Organização Nacional da Indústria do Petróleo (onip) (Rodrigues Alonso, 2013). Además, Prominp cuenta con un Comité Ejecutivo, Comités Sectoriales y con un Coordinador Ejecutivo que hace parte del Ministerio de Minas y Energía (Rodrigues Alonso, 2013).

22 Ver la historia de Prominp en http://www.prominp.com.br/prominp/pt_br/conteudo/sobre-o-prominp.htm

23 Según Prominp: "Desde la primera ronda de licitaciones en 1999, la Agencia Nacional del Petróleo, Gas Natural y Biocombustibles (ANP) establece los requisitos mínimos para el contenido local en sus contratos de concesión con los operadores ganadores de las inversiones en las fases de exploración y desarrollo de producción”. Ver: http:// www.prominp.com.br/prominp/pt_br/conteudo/conteudo-local.htm 
ellos requieran priorizar determinadas regiones con mayor potencial. En segundo lugar, más que la simple formulación de políticas públicas, superar estos cuellos de botella requiere un trabajo estrecho entre el Estado, el sector extractivo, el sector industrial y de servicios y las agremiaciones empresariales. Además, como explican Tordo et al. (2013), la promoción del "contenido local" en el sector extractivo depende de la consistencia (y coordinación) entre las políticas que buscan incentivar los encadenamientos locales y las políticas de desarrollo (p. ej., fortalecimiento del capital humano) y de competitividad (p. ej., mejoramiento de infraestructura).

No obstante lo anterior, Colombia está en mora de contar con una política sectorial que pretenda desarrollar las capacidades de los proveedores locales para generar encadenamientos con el sector extractivo. Esto, podrá tener un mayor nivel de intervención estatal (tipo Cadena y Pinzón, 2011), o un menor nivel de intervención estatal (tipo Martínez y Aguilar, 2013), pero en todo caso algún grado de interacción entre los ámbitos público y privado.

\section{REFERENCIAS}

ANDI (2009). La cadena de hidrocarburos: una oportunidad para la industria Colombiana. Resumen Ejecutivo. Bogotá: ANDI.

Blomströм, M. у Кокко, A. (2007). From Natural Resources to High-Tech Production: The Evolution of Industrial Competitiveness in Sweden and Finland. En Lederman, D. y Maloney, W. F. (eds.). Natural Resources: Neither Curse nor Destiny (pp. 213-256). Washington, DC: Stanford University Press and The World Bank. Cadena, A. y Pinzón, W. (2011). Clusters minero energéticos en Colombia: desarrollo, hallazgos y propuestas. Revista de Ingeniería, 34, 49-60.

Castillo, O. (2014). El sector minero colombiano en el futuro: potencial y competitividad por mineral, transformaciones y encadenamientos productivos. En Benavides (comp.). Insumos para el desarrollo del Plan Nacional de Ordenamiento Minero (pp. 69-102). Bogotá: Universidad de los Andes, CIDER, Ediciones Uniandes, Unidad de Planeación Minero Energética (UPME).

Castro, F., Zuleta, L. A., Alandette, J. F., FonseCa, S. M. y Martínez, S. (2012). Dinámica regional del sector de bienes y servicios petroleros en Colombia, Nueva Serie Cuadernos de Fedesarrollo, 41.

Consejo Minero (2013). Buenas prácticas en tiempos de cambio. En Ministerio de Minería y la Comisión Chilena del Cobre (coord.). Minería en Chile: impacto en regiones y desafios para su desarrollo (pp. 58-83). Santiago: Ministerio de Minería y la Comisión Chilena del Cobre.

Comisión Chilena del Cobre (2013). Efecto macroeconómico y el aporte al desarrollo regional. En Ministerio de Minería y la Comisión Chilena del Cobre (coord.) Minería en Chile: Impacto en Regiones y Desafíos para su Desarrollo (pp. 37-57). Santiago: Ministerio de Minería y la Comisión Chilena del Cobre.

de Oliveira, R. (2015). Local Content Requirements in the Oil and Gas Industry in Developing Countries: The Return of IsI? (Septiembre 8, 2015). мiт Political Science Department Research Paper, 20.

Esteves, A. M., Coyne, B y Moreno, A. (2013). Iniciativas sobre contenido local: mejorar los beneficios 
subnacionales de los sectores petrolero, gasífero y minero. Revenue Watch Institute. Recuperado de www.revenuewatch.org

Fundación Chile (2013). El impacto de la industria minera en empresas proveedoras locales. En Ministerio de Minería y la Comisión Chilena del Cobre (coord.) Minería en Chile: impacto en regiones y desafios para su desarrollo (pp. 139-156). Santiago: Ministerio de Minería y la Comisión Chilena del Cobre.

Gelb, A. (2011). Economic Diversification in Resource Rich Countries. En Arezki, R., Gylfason, T. y Sy, A. (eds.). Beyond the curse: policies to harness the power of natural resources (pp. 55-80). Washington: International Monetary Fund.

GüEsGuán, O. (2015). El fin del frenesí petrolero. El Espectador, 15 de febrero. Recuperado de http:// www.elespectador.com/noticias/economia/elfin-del-frenesi-petrolero-articulo-544708

Gylfason, T. (2011). Natural resource endowment: A mixed blessing? En Arezki, R., Gylfason, T. y Sy, A. (eds.). Beyond the curse: policies to harness the power of natural resources (pp. 7-34). Washington: International Monetary Fund.

HARTWICK, J. M. (1977). Intergenerational equity and the investing of rents from exhaustible resources. The American Economic Review, 67 (5), 972-974. Recuperado de http://www.jstor.org/ stable/1828079

Herzer D. y Nowak-Lehmann, F. (2004). Export Diversification, Externalities and Growth. Discussion Papers, 99. Gottingen: Ibero-America Institute for Economic Research.

Humphreys, M., Sachs, J. y Stiglitz, J. (2007). Introduction: What Is the Problem with Natural Resource Wealth? En Humphreys, S. y Stiglitz, J. (eds.). Escaping the Resource Curse. New York: Columbia University Press.
Instituto Peruano de Economía (2012). Efecto de la minería sobre el empleo, el producto y recaudación en Perú. Recuperado de http://ipe.org.pe/documentos/efecto-de-la-mineria-sobre-el-empleo-elproducto-y-la-recaudacion-en-el-peru-0

LAFUente, J. (2015). La burbuja del petróleo estalla en Puerto Gaitán. El Espectador, 4 de agosto. Recuperado de http://www.elespectador.com/ noticias/economia/burbuja-del-petroleo-estallapuerto-gaitan-articulo-577184

Leahy, J. (2015). Brazil: Oily mess. Financial Times, mayo 14. Recuperado de http://www.ft.com/ $\mathrm{cms} / \mathrm{s} / 0 /$ fc43ddc6-f94f-11e4-be7b-00144feab7de.html?siteedition=uk\#slide0

Macroconsult (2012). Impacto económico de la minería en el Perú. Recuperado de http://www. cimu.org.uy/wp-content/uploads/18-Impactoecomonico-de-actividad-minera-en-el-PeruJunio-2012.pdf

Martínez Ortiz, A. y Aguilar Londoño, T. (2013). Estudio sobre los impactos socioeconómicos del sector minero en Colombia: encadenamientos sectoriales. Nueva Serie - Cuadernos de Fedesarrollo, 47.

Ministério de Minas e Energía (2013). Análise da Evolução de Política Governamental de Conteúdo Local no Brasil e perspectivas para os próximos anos. Presentación en el $10^{\circ}$ Encontro Nacional Do Prominp. Recuperado de http://www.prominp.com.br/lumis/portal/file/fileDownload. jsp?fileId=8A9D2A9742A04E14014310F2 7D051709

Moreno, J. (2015). Petroleras se reacomodan en el Llano. El Espectador, 8 junio. Recuperado de http://www.elespectador.com/noticias/econo$\mathrm{mia} /$ petroleras-se-reacomodan-el-llano-articulo-565251 
Olivera, M., Zuleta, L. A., Aguilar, T. y Orozco, A. (2011). Impacto del sector de servicios petroleros en la economía colombiana. Nueva Serie Cuadernos de Fedesarrollo, 36.

Perry, G. y Palacios, C. (2013). Emprendimiento alrededor del Sector de la Minería y el Petróleo en Colombia, Documentos CEDE, 13. Bogotá: Universidad de los Andes.

Raval, A. y Sheppard, D. (2015). Global oil supply grows at breakneck speed, says IEA. Financial Times, agosto 12. Recuperado de http://www. ft.com/intl/cms/s/0/f102aa60-40c5-11e5-9abe5b335da3a90e.html

Reina, M. y Cárdenas, M. (2008). La minería en Colombia: impacto socioeconómico y fiscal. Nueva Serie Cuadernos de Fedesarrollo, 25.

RYGGVIK, H. (2013). Building a skilled national offshore oil industry: The Norwegian experience. Oslo: NHO.

Rodrigues Alonso, P. S. (2013). Resultados do Prominp. Presentación en el $10^{\circ}$ Encontro Nacional Do Prominp. Recuperado de http://www.prominp.com.br/lumis/portal/file/fileDownload. jsp?fileId=8A9D2A9742A04E14014310118 CC80113

SEgal, D. (2015). Petrobras Oil Scandal Leaves Brazilians Lamenting a Lost Dream. En The New
York Times, agosto 7. Recuperado de http://www. nytimes.com/2015/08/09/business/international/effects-of-petrobras-scandal-leave-brazilianslamenting-a-lost-dream.html?_r=0

Tordo, S., Warner, M., Manzano, O. E. y Anouti, Y. (2013). Local Content Policies in the Oil and Gas Sector. World Bank Study. Washington: World Bank. doi:10.1596/978-0-8213-9931-6.

van der Ploeg, F. y Venables, A. J. (2012). Natural resource wealth: The challenge of managing a windfall. Oxford: Centre for the Analysis of Resource Rich Economies, University of Oxford.

World Bank (2006). Where is the Wealth of $\mathrm{Na}$ tions? Measuring capital for the 21th century. Washington: The World Bank. Recuperado de http://siteresources.worldbank.org/INTE EI/214578-1110886258964/20748034/All.pdf

Wright, G. y Czelusta, J. (2002). Exorcizing the Resource Curse: Minerals as a Knowledge Industry, Past and Present. Working Paper, Stanford University.

Vega, C. (2015). Primeros efectos de la caída del precio del petróleo. El Espectador, 21 de enero. Recuperado de http://www.elespectador.com/noticias/ economia/primeros-efectos-de-caida-del-preciodel-petroleo-articulo-539063 